\title{
Aplicação das Dietary Reference Intakes na avaliação da ingestão de nutrientes para indivíduos
}

\author{
Application of Dietary Reference Intakes for \\ assessment of individuals
}

Dirce Maria Lobo MARCHIONI

Betzabeth SLATER ${ }^{2}$

Regina Mara FISBERG²

\section{R E S U M O}

A avaliação do estado nutricional é uma das práticas clínicas fundamentais para tomar-se a decisão quanto ao diagnóstico nutricional de um indivíduo e à conduta dietética a ser-lhe prescrita. A adequação da ingestão de nutrientes é um dos componentes da avaliação nutricional e é feita a partir de valores de referência que se constituem em estimativas das necessidades fisiológicas desses nutrientes e metas de ingestão dos mesmos. Colocam-se hoje à disposição dos profissionais um novo conjunto de valores de referência que constituem um avanço importante no modo de interpretar a adequação dietética: as Dietary Reference Intakes. Este artigo aborda os métodos propostos para avaliação da adequação da ingestão de nutriente às necessidades do indivíduo, utilizando os novos valores de referências.

Termos de indexação: políticas de nutrição, ingestão de alimentos, avaliação nutricional.

\section{A B S T R A C T}

The nutritional status assessment is one of the fundamental clinical approaches in making a decision about nutritional diagnosis and dietetic behavior, in order to prescribe an adequate diet therapy. The evaluation of nutrient intakes is a component of the nutritional assessment and it is made from estimates of nutrient physiological needs and goals for good nutrition, known as reference values. A new group of reference values is recently available for health professionals: the Dietary Reference Intakes, which represent an important progress in the field of dietary assessment interpretation. This paper discusses the proposed methods for the individual nutrient intake assessment, using the Dietary Reference Intakes.

Index terms: nutrition policy, eating, nutrition assessment.

\footnotetext{
1 Doutoranda, Departamento de Nutrição, Faculdade de Saúde Pública, Universidade de São Paulo.

2 Departamento de Nutrição, Faculdade de Saúde Pública, Universidade de São Paulo. Av. Dr. Arnaldo 715, 01246-904, Cerqueira César, São Paulo, SP, Brasil. Correspondência para/Correspondence to: R.M. FISBERG. E-mail: rfisberg@usp.br
} 


\section{N T R O D U Ç Ã O}

O estado nutricional reflete o grau no qual as necessidades fisiológicas dos nutrientes estão sendo alcançadas, ou seja, a relação entre o consumo de alimentos e as necessidades nutricionais do indivíduo'.

A avaliação nutricional pretende detectar problemas nutricionais, colaborando para a promoção ou recuperação da saúde. Na prática clínica utiliza-se a análise da história clínica, dietética e social, dados antropométricos, dados bioquímicos e interação entre drogas e nutrientes para estabelecer o diagnóstico nutricional e servir de base para o planejamento e orientação dietética ${ }^{2}$. A avaliação da ingestão de nutrientes é parte da avaliação nutricional, sendo utilizada para a tomada de decisão quanto à adequação do consumo alimentar do indivíduo e auxiliar no estabelecimento da conduta dietoterápica, em conjunto com os outros parâmetros citados.

Este artigo pretende abordar os métodos propostos para avaliação da adequação da ingestão de nutrientes, por um indivíduo, utilizando os valores de referência Dietary Reference Intakes ${ }^{3}$.

\section{O que são as DRI}

Relativamente novas no campo da Nutrição, as DRI definem-se como um conjunto de quatro valores de referência de ingestão de nutrientes, estabelecidos e usados para o planejamento e avaliação das dietas do indivíduo ou grupos de indivíduos saudáveis, segundo estágio de vida e gênero ${ }^{3}$.

Estes valores incorporam as recomendações dietéticas Recommended Dietary Allowance (RDA) e foram desenvolvidos para a população americana e canadense. Para sua formulação, foram formados Comitês de Especialistas pelo Food and Nutrition Board, pelo Institute of Medicine da National Academy of Sciences dos Estados Unidos e pelo Health Canadá. Este órgão tem publicado os relatórios dos Comitês, que estão disponíveis para consulta na Internet, no site www.nap.edu.org. A seguir, são definidos os valores de referência propostos:

\section{Estimated Average Requirement (EAR)}

É o valor médio de ingestão diária estimada para atender às necessidades de $50 \%$ de indivíduos saudáveis de um grupo em determinado estágio de vida e gênero. Neste nível de ingestão, a outra metade do grupo não tem suas necessidades atingidas (Figura 1).

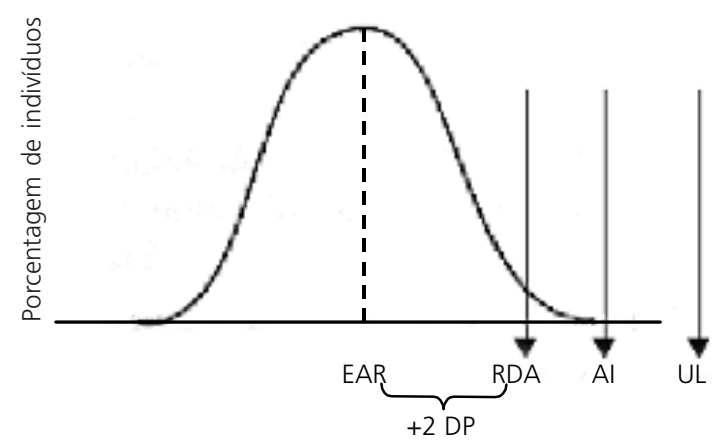

Distribuição da necessidade média do nutriente

(*) EAR: Necessidade média estimada; RDA: Ingestão dietética recomendada; Al: Ingestão adequada; UL Nível máximo tolerável de ingestão.

Figura1. Modelo para os valores de referência da dieta.

A EAR é baseada em um critério específico de adequação e formulada a partir de uma revisão cuidadosa da literatura. Na seleção deste critério é considerada a redução do risco de doenças por deficiência ou excesso, juntamente com outros parâmetros de saúde.

Aplica-se a EAR, juntamente com a estimativa da variabilidade da necessidade do nutriente, para avaliar a dieta, tanto de indivíduos quanto de grupos de indivíduos e também para calcular a $\operatorname{RDA}^{3}$.

\section{Recommended Dietary Allowance (RDA)}

Considerada como o nível de ingestão diária, a RDA é a quantidade do nutriente sufi- 
ciente para atender à necessidade de aproximadamente $97 \%$ a $98 \%$ dos indivíduos saudáveis de um grupo em determinado estágio de vida e gênero.

A RDA é derivada matematicamente a partir da EAR e do desvio-padrão da necessidade do nutriente sob a premissa de normalidade da necessidade do nutriente (ou seja, a distribuição é simétrica em torno da média, e a média e mediana são iguais). A RDA é definida como o valor correspondente a dois desvios padrão acima da necessidade média $(E A R): \quad R D A=E A R+2 D P_{\text {necessidade }}$

Se não houver dados suficientes para estimar o desvio-padrão da ingestão, ou se o desvio-padrão relatado na literatura for inconsistente, assume-se um coeficiente de variação ( $C V$ = desvio-padrão da necessidade/necessidade média $X 100$ ) teórico de $10 \%$ para a maioria dos nutrientes ${ }^{3}$. Nessa circunstância: RDA = 1,2 XEAR.

\section{Adequate Intake (AI)}

Na situação de insuficiência da informação para estabelecer a necessidade média estimada do nutriente (EAR), e portanto, a RDA, foi proposto pelo Comitê um valor de Al. A Al é baseada em níveis de ingestão derivados experimentalmente ou por aproximações da média de ingestão do nutriente por um grupo (ou grupos) de indivíduos aparentemente saudáveis, que mantêm um estado nutricional definido ou determinado critério de adequação. Exemplos de estado nutricional definido, são: crescimento normal, manutenção de níveis normais de nutrientes no plasma, e outros aspectos de adequação nutricional ou estado geral de saúde. É usada, quando não é possível determinar a RDA, como uma meta de ingestão do nutriente para os indivíduos.

Espera-se que a Al exceda a RDA para um critério específico de adequação. Na ausência de RDA, a Al é utilizada como meta de ingestão individual. No entanto, a sua utilização para a avaliação de dietas é limitada ${ }^{3}$.

\section{Tolerable Upper Intake Level (UL)}

O UL é o mais alto nível de ingestão habitual do nutriente que provavelmente não coloca em risco de efeitos adversos quase todos os indivíduos em um determinado estágio de vida e gênero. À medida que a ingestão aumenta acima do UL, aumenta o risco potencial de efeitos prejudiciais à saúde. É um nível de ingestão com alta probabilidade de ser tolerado biologicamente, mas não um nível recomendado de ingestão. $\mathrm{Na}$ utilização do UL para averiguar a suspeita de ingestão excessiva do nutriente, os profissionais devem levar em conta vários parâmetros, tais como: fonte do nutriente, estado fisiológico do indivíduo e período de tempo de ingestão habitual elevada do nutriente ${ }^{3}$.

\section{Seleção das DRI para avaliação da adequação da dieta}

Quando um valor de EAR para um nutriente estiver disponível, este é o valor que deve ser utilizado para fazer uma estimativa quantitativa da adequação da ingestão habitual do nutriente. Apesar da RDA ser a meta de ingestão individual, não é recomendado seu uso para averiguar esta adequação. Quando somente um valor de $\mathrm{Al}$ estiver disponível, é possível tão somente determinar quantitativamente se a ingestão habitual está acima da Al, com determinado nível de confiança. No entanto, nenhuma conclusão pode ser feita se a ingestão habitual estiver abaixo da Al. É possível, também, determinar se a ingestão do nutriente excede a UL, como veremos adiante $^{2}$

Deve-se ressaltar que a estimativa de ingestão de nutrientes não pode ser usada isoladamente para avaliar o estado nutricional dos indivíduos. Se na avaliação da ingestão habitual do nutriente houver indicações de inadequação, recomenda-se que sejam feitas avaliações clínicas ou bioquímicas complementares do estado nutricional do indivíduo. 


\section{Adequação da ingestão alimentar}

Para avaliar a ingestão de nutrientes é necessário inicialmente estabelecer a ingestão habitual do indivíduo e, em seguida, confrontá-la com as necessidades deste mesmo indivíduo.

A ingestão habitual é definida como a média de ingestão do nutriente por um grande período de tempo. De acordo com esta definição, só poderá ser avaliada por um grande número de dias. Este variará segundo os nutrientes a serem estudados. Uma estimativa correta da dieta habitual do indivíduo envolve o conhecimento da variabilidade intrapessoal e a escolha de um método sensível para estimar o consumo. Assinala-se que não há método de avaliação de ingestão alimentar livre de erro, nem é factível obter estimativas de ingestão de um grande número de dias, por problemas de tempo e custo ${ }^{4-6}$.

Por outro lado, a necessidade é definida como o menor valor de ingestão continuada do nutriente que irá manter um nível definido de nutrição em um indivíduo, para um dado critério de adequação nutricional. É evidente que a determinação da necessidade de um indivíduo exigiria um ambiente de laboratório, onde o sujeito fosse alimentado com doses variáveis do nutriente estudado durante um período de tempo, e, concomitantemente, fossem feitas numerosas medidas bioquímicas e fisiológicas. Dessa forma, podemos concluir que não é possível determinar, com acurácia, nem o verdadeiro consumo habitual nem a verdadeira necessidade do nutriente em um determinado indivíduo. Apesar disso, é possível avaliar aproximadamente se a ingestão de um indivíduo atinge as necessidades. Esta avaliação pode ser chamada de Adequação Aparente ${ }^{2}$.

Para verificar a adequação aparente da ingestão do nutriente, é necessária a obtenção das seguintes informações: a estimativa da ingestão dietética total, incluindo alimentos, suplementos e, eventualmente, teor mineral da água e a estimativa das necessidades do indivíduo.

\section{Estimativa da ingestão dietética}

Para estabelecer a ingestão de nutrientes devem ser utilizados métodos de inquérito alimentar, sendo os mais indicados os registros alimentares e recordatório de 24 horas. A melhor estimativa da ingestão do nutriente pelo indivíduo é dada pela média de vários dias de consumo dietético obtido por estes métodos. Os métodos como história dietética e o questionário de freqüência alimentar, que se utilizam de listas fechadas de alimentos, não são apropriados para esta finalidade.

Além disso, é necessário procurar-se estabelecer o padrão habitual de consumo alimentar, reconhecendo-se que este é um grande desafio, uma vez que há variabilidade intrapessoal. Deve-se ter especial atenção com o consumo de alimentos que são fontes de determinados nutrientes e que não são consumidos diariamente $^{7}$. Como pode ser percebido facilmente, a medida da dieta feita por meio do registro de um único dia, como é feito no recordatório de 24 horas (R24h), não tem esta propriedade. Sugere-se a utilização do registro de 3 ou mais dias, ou, como alternativa, a aplicação de vários R24h. Deve-se aplicar quaisquer métodos selecionados, em dias alternados e abrangendo um dia de final de semana ${ }^{8}$.

Não considerar a variação da ingestão de nutrientes devido à variabilidade do consumo alimentar pode levar à subestimação ou superestimação da ingestão habitual. Dessa forma, é fundamental obter-se uma estimativa da variabilidade do consumo intrapessoal, que é o componente que explica a variação do consumo de alimentos do indivíduo no dia a dia ${ }^{9}$. Embora, ao avaliarmos dois ou mais dias, tenhamos uma medida de variabilidade intrapessoal, o "Subcomitê para Uso e Interpretação das DRIs" recomenda que seja utilizada a estimativa desta variabilidade obtida em estudos de consumo alimentar em populações (Tabelas 1 e 2). No Brasil, não temos disponíveis dados de base populacional sobre a variabilidade do consumo intrapessoal. 
Portanto, a única alternativa, até o presente, é a utilização dos dados americanos².

\section{Estimativa das necessidades do indivíduo}

A melhor estimativa das necessidades do indivíduo é dada pela EAR, já que, como foi discutido, não se conhece a necessidade verdadeira do indivíduo que se está avaliando. Obviamente, há uma variação da necessidade entre os indivíduos, mesmo sendo estes pertencentes ao mesmo estágio de vida e gênero. Assim, é importante levar-se em conta esta variabilidade, que é dada pelo coeficiente de variação (CV) do nutriente. Para a maioria dos nutrientes, foi assumido uma variação de 10\%, com exceção da niacina, cujo CV foi estabelecido como $15 \%^{2,10}$.

\section{Calculando a adequação aparente}

De posse das informações necessárias: as estimativas da ingestão e da variabilidade da ingestão do nutriente; as estimativas da necessidade (EAR) e da variação da necessidade (CV) do nutriente, passa-se a calcular a adequação aparente. Para isso, desenvolveu-se uma abordagem estatística que permite estimar o grau de confiança com que a ingestão do nutriente alcança a necessidade do indivíduo. Esta abordagem compara a diferença entre a ingestão relatada (a melhor estimativa da ingestão habitual) e a EAR. A equação desenvolvida também leva em conta a variabilidade da necessidade e a variação intrapessoal (do dia a dia). O resultado é um escore-Z, por meio do qual determina-se a probabilidade da dieta estar adequada, ou seja, o grau de confiança que a ingestão alcança as necessidades ${ }^{2}$.

Tabela 1. Estimativas de desvio-padrão intrapessoal para vitaminas e minerais baseadas no Continuing Survey of Food Intakes by Individuals, 1994-1996 em mulheres de diferentes faixas etárias.

\begin{tabular}{|c|c|c|c|c|}
\hline \multirow{2}{*}{ Sexo feminino } & \multicolumn{4}{|c|}{ Estimativa de desvio-padrão intrapessoal $\left(\mathrm{dp}_{\text {intr }}\right)$} \\
\hline & Crianças 4 a 8 anos & Adolescentes 9 a 18 anos & Adultos 19 a 50 anos & Adultos $>51$ anos \\
\hline Vitamina $A^{*}(\mu \mathrm{g})$ & 808 & 852 & 1300 & 1255 \\
\hline Caroteno $(\mathrm{RE})^{\star}$ & 452 & 549 & 799 & 796 \\
\hline Vitamina $E(m g)^{*}$ & 3 & 4 & 5 & 6 \\
\hline Vitamina $C(m g)^{*}$ & 61 & 81 & 73 & 61 \\
\hline Tiamina (mg) & 0,5 & 0,6 & 0,6 & 0,5 \\
\hline Riboflavina (mg) & 0,6 & 0,7 & 0,6 & 0,6 \\
\hline Niacina (mg) & 6 & 8 & 9 & 7 \\
\hline Vitamina $B_{6}(\mathrm{mg})$ & 0,6 & 0,7 & 0,8 & 0,6 \\
\hline Folato $(\mu \mathrm{g})$ & 99 & 128 & 131 & 12 \\
\hline Vitamina $B_{12} *(\mu \mathrm{g})$ & 9,6 & 5,5 & 12 & 10 \\
\hline Cálcio (mg) & 313 & 374 & 325 & 256 \\
\hline Fósforo (mg) & 321 & 410 & 395 & 313 \\
\hline Magnésio (mg) & 61 & 86 & 86 & 74 \\
\hline Ferro $(\mathrm{mg})$ & 5 & 6 & 7 & 5 \\
\hline Zinco(mg) & 3 & 5 & 6 & 5 \\
\hline Cobre (mg) & 0,4 & 0,5 & 0,6 & 0,5 \\
\hline Sódio (mg) & 930 & 1313 & 1839 & 1016 \\
\hline Potássio (mg) & 631 & 866 & 851 & 723 \\
\hline
\end{tabular}

(*) Nutrientes com CV > 60\% a $70 \%$.

Fonte: Tabela adaptada do IDM (2000)². 
Tabela 2. Estimativas de desvio-padrão intrapessoal para vitaminas e minerais baseadas no Continuing Survey of Food Intakes by Individuals, 1994-1996 em homens de diferentes faixas etárias.

\begin{tabular}{|c|c|c|c|c|}
\hline \multirow{2}{*}{ Sexo feminino } & \multicolumn{4}{|c|}{ Estimativa de desvio-padrão intrapessoal $\left(\mathrm{dp}_{\text {intr }}\right)$} \\
\hline & Crianças 4 a 8 anos & Adolescentes 9 a 18 anos & Adultos 19 a 50 anos & Adultos $>51$ anos \\
\hline Vitamina $A^{*}(\mu \mathrm{g})$ & 723 & 898 & 1160 & 1619 \\
\hline Caroteno $(\mathrm{RE})^{*}$ & 454 & 681 & 875 & 919 \\
\hline Vitamina $\mathrm{E}(\mathrm{mg})^{*}$ & 3 & 5 & 7 & 9 \\
\hline Vitamina $C(\mathrm{mg})^{*}$ & 74 & 93 & 93 & 72 \\
\hline Tiamina (mg) & 0,5 & 0,8 & 0,9 & 0,7 \\
\hline Riboflavina (mg) & 0,7 & 1,0 & 1,0 & 0,8 \\
\hline Niacina (mg) & 7 & 11 & 12 & 9 \\
\hline Vitamina $B_{6}(\mathrm{mg})$ & 0,7 & 1,0 & 1,0 & 0,8 \\
\hline Folato $(\mu \mathrm{g})$ & 117 & 176 & 180 & 150 \\
\hline Vitamina $B_{12}^{*}(\mu \mathrm{g})$ & 4,7 & 5,0 & 13 & 14 \\
\hline Cálcio (mg) & 353 & 505 & 492 & 339 \\
\hline Fósforo (mg) & 352 & 542 & 573 & 408 \\
\hline Magnésio (mg) & 71 & 109 & 122 & 94 \\
\hline Ferro (mg) & 6 & 9 & 9 & 7 \\
\hline Zinco (mg) & 4 & 8 & 9 & 8 \\
\hline Cobre (mg) & 0,4 & 0,6 & 0,7 & 0,7 \\
\hline Sódio (mg) & 957 & 1630 & 1819 & 1323 \\
\hline Potássio (mg) & 750 & 1130 & 1147 & 922 \\
\hline
\end{tabular}

(*) Nutrientes com CV > 60\% a $70 \%$.

Fonte: Tabela adaptada do IDM (2000)².

$$
\mathrm{z}=\mathrm{D} / \mathrm{DP} \mathrm{D}_{\mathrm{D}}=\frac{\bar{y}-E A R}{\sqrt{V_{\text {nec }}+\left(V_{\mathrm{int}} / n\right)}} \quad \text { (equação 1) }
$$

Onde:

$\bar{\gamma}$ é a média de ingestão de $\mathrm{n}$ dias do nutriente pelo indivíduo

EAR é a melhor estimativa da necessidade do nutriente pelo indivíduo

$V_{\text {nec }}$ é a variância da necessidade

$V_{\text {int }}$ é a variância intrapessoal

Ambas as variâncias são computadas como o quadrado dos desvios-padrão correspondentes, sendo que o $n$ corresponde ao número de dias em que o indivíduo teve sua ingestão avaliada.

Para exemplificar, suponhamos uma mulher de 20 anos, cuja ingestão média diária de riboflavina, obtida por meio de um registro alimentar de três dias, foi de 1,2mg. A EAR para este nutriente é de $0,9 \mathrm{mg} / \mathrm{d}$. Identificando os termos da equação temos:

$$
\begin{aligned}
& \bar{\gamma}=1,2 \mathrm{mg} \\
& \mathrm{EAR}=0,9 \mathrm{mg} \\
& \mathrm{V}_{\text {nec }}=\left(\mathrm{dp}_{\text {nec }}\right)^{2} \text {, temos que o desvio-padrão }
\end{aligned}
$$
da necessidade corresponde a $10 \%$ da EAR, portanto:

$$
\begin{aligned}
& V_{\text {nec }}=(0,1 * 0,9)^{2}=0,0081 \\
& V_{\text {int }}=\left(d p_{\text {int }}\right)^{2} \text {, observando que o desvio- }
\end{aligned}
$$
-padrão corresponde à variação intrapessoal é obtido a partir de estudos populacionais americanos, conforme pode ser observado nas tabelas 1 e 2. Portanto, o desvio-padrão para riboflavina é de $0,6 \mathrm{mg}$. Assim, temos:

$$
\begin{aligned}
& V_{\text {int }}=(0,6)^{2}=0,36 \\
& n=3 \text {, correspondendo a três dias de registro }
\end{aligned}
$$
alimentar. 
Substituindo estes termos na equação 1, obtemos:

$$
z=D / D P_{D}=\frac{1,2-0,9}{\sqrt{0,0081+(0,36 / 3}}=0,838
$$

Na tabela de valores de $Z$, este valor corresponde a uma área que indica a probabilidade de aproximadamente $85 \%$. Como conclusão, podemos dizer que a dieta está adequada, com $85 \%$ de confiabilidade ${ }^{2}$.

Devemos notar que a RDA para este nutriente é de 1,1 mg/dia. Assim, na abordagem convencional diríamos que o indivíduo ingeriu aproximadamente $110 \%$ da RDA, mas, neste nível de consumo, avaliado em 3 dias, há ainda a probabilidade de $15 \%$ deste consumo estar inadequado.

Se prefixarmos um determinado nível de confiabilidade, por exemplo $90 \%$, notaremos que o valor de ingestão para o nutriente diminui à medida que aumenta o número de dias avaliados.

\section{Considerações de ordem prática}

Apesar de não haver programas de computador que analisem a dieta utilizando esta nova abordagem, estes deverão estar disponíveis em breve. Enquanto isto não ocorre, pode-se usar alguns recursos para facilitar os profissionais da saúde no cálculo da adequação da dieta. Pode-se prefixar o nível de confiança que se acredita desejável para considerar a dieta adequada, por exemplo, $85 \%$, $90 \%$ ou $97,5 \%$. Também pode-se padronizar os métodos de inquérito que serão utilizados na prática clínica, tais como: registros alimentares de três dias, ou pelo menos dois recordatórios de 24 horas.

Pode-se observar (Tabela 3) quais seriam os valores de ingestão de diversos nutrientes, que atendem às premissas do método, prefixando-se os valores de confiabilidade, considerando-se três dias de inquérito.

Também, como uma forma prática sugerida pelo Comitê, se a ingestão do nutriente for menor que a EAR, esta deve ser implementada; se a ingestão estiver entre a EAR e a RDA, há risco de inadequação, e provavelmente a ingestão deva ser aumentada; e se a ingestão estiver acima da RDA, e, ao mesmo tempo, um número expressivo de dias tiver sido avaliado, então é pouco provável que a ingestão seja inadequada.

Tabela 3. Valores de ingestão do nutriente segundo nível de confiança, para homens e mulheres na faixa etária de 19 a 50 anos, com base em três dias de inquérito alimentar.

\begin{tabular}{|c|c|c|c|c|c|c|c|c|}
\hline & \multicolumn{4}{|c|}{ Mulhres } & \multicolumn{4}{|c|}{ Homens } \\
\hline & \multicolumn{4}{|c|}{ Confiabilidade de adequação } & \multicolumn{4}{|c|}{ Confiabilidade de adequação } \\
\hline & $85 \%$ & $90 \%$ & $95 \%$ & $98 \%$ & $85 \%$ & $90 \%$ & $95 \%$ & $98 \%$ \\
\hline Tiamina $(\mathrm{mg})^{*}$ & 1,26 & 1,35 & 1,49 & 1,62 & 1,53 & 1,66 & 1,87 & 2,06 \\
\hline Riboflavina $(\mathrm{mg})^{*}$ & 1,26 & 1,35 & 1,49 & 1,62 & 1,69 & 1,83 & 2,07 & 2,28 \\
\hline Niacina $(m g)^{*}$ & 16,45 & 17,81 & 20,00 & 21,90 & 19,16 & 20,95 & 23,81 & 26,32 \\
\hline Vit $B_{6}(m g)^{*}$ & 1,57 & 1,69 & 1,88 & 2,05 & 1,69 & 1,83 & 2,07 & 2,28 \\
\hline Folato $(\mathrm{mcg})^{*}$ & 402 & 423 & 456 & 484 & 429 & 456 & 499 & 537 \\
\hline Fósforo $(\mathrm{mg})^{*}$ & 815 & 874 & 968 & 1050 & 916 & 1000 & 1134 & 1252 \\
\hline Magnésio $(\mathrm{mg})^{\star *}$ & 311 & 325 & 347 & 367 & 408 & 427 & 458 & 459 \\
\hline Manganês $(\mathrm{mg})^{* * *}$ & 321 & 335 & 358 & 378 & 429 & 448 & 480 & 507 \\
\hline
\end{tabular}

(*) faixa etária: 19 a 50 anos; (**) faixa etária 19 a 30 anos; (***) faixa etária de 31 a 50 anos. 


\section{A AI na avaliação de indivíduos}

A EAR não foi ainda estabelecida para todos os nutrientes. Nestes casos, uma Al é o valor de referência disponível, e, para estes nutrientes, o método discutido acima para estimar a adequação aparente de ingestão não pode ser utilizado. Chama-se a atenção para a diferença entre os valores de referência. A EAR representa a mediana da necessidade do nutriente em determinado estágio de vida e gênero e, por definição, uma ingestão neste nível pode ser inadequada para metade do grupo. Em contraste, a Al representa uma ingestão (não necessidade) que provavelmente excede a verdadeira, mas desconhecida, necessidade de quase todos os indivíduos saudáveis dentro de um determinado gênero e estágio de vida. Neste sentido, é análoga à RDA. Quando se compara a ingestão com a Al, o que se pode concluir é se a ingestão está acima deste valor ou não. Se a ingestão habitual do nutriente estiver acima da Al, estará certamente adequada. Os valores de ingestão do nutriente abaixo da Al, por outro lado, ainda estarão adequados para um grupo de pessoas. No entanto, nenhuma conclusão quantitativa pode ser feita.

Apesar destas considerações, pode-se testar se a ingestão habitual do indivíduo excede a Al e, como decorrência, concluir que o consumo está adequado. O teste é similar ao apresentado, considerando-se igualmente a variabilidade intrapessoal, dividida pela raiz quadrada do número de dias em que o indivíduo foi avaliado².

$$
\mathrm{z}=\quad \begin{array}{lll} 
& A I & \text { (equação 2) }
\end{array}
$$

Onde:

$\bar{\gamma}$ é a média de ingestão de $\mathrm{n}$ dias do nutriente pelo indivíduo

Al é o valor de referência estabelecido na impossibilidade de estabelecer a EAR pelo indivíduo

$d p_{\text {int }}$ é a o desvio-padrão intrapessoal, obtido em estudos populacionais $n$ corresponde ao número de dias em que o indivíduo teve sua ingestão avaliada

Este método se mostra inadequado quando a distribuição dos valores de ingestão do nutriente for assimétrica, o que pode ser identificado quando o CV for maior que $60 \%$ a $70 \%$.

\section{O UL na avaliação de indivíduos}

Quando um indivíduo esta ingerindo quantidades excessivas do nutriente é possível avaliar a probabilidade de risco da ocorrência de efeitos adversos. Ressalta-se que a UL para alguns nutrientes refere-se somente à ingestão de suplementos, medicamentos e alimentos fortificados, enquanto para outros todas as fontes foram consideradas. No caso da UL, a ingestão crônica é objeto de preocupação, já que uma ingestão eventual neste nível, ou pouco acima, não é motivo de alarme. Um cálculo similar ao feito para a Al pode ser utilizado para verificar se a ingestão habitual do nutriente excede a UL. O escore-Z é comparado com os valores de referência e pode-se concluir com que grau de confiabilidade a ingestão é excessiva ou segura².

$$
\mathrm{z}=\frac{\bar{y}-U L}{d p_{\text {int }} / \sqrt{n}} \text { (equação 3) }
$$

Onde:

$\bar{\gamma}$ é média de ingestão de $\mathrm{n}$ dias do nutriente pelo indivíduo

UL é o valor de referência estabelecido como limite superior de ingestão do nutriente que não causa efeitos adversos

$d_{\text {int }}$ é a o desvio-padrão intrapessoal, obtido em estudos populacionais

$n$ corresponde ao número de dias em que o indivíduo teve sua ingestão avaliada

Este método também se mostra inadequado quando a distribuição dos valores de 
ingestão do nutriente for assimétrica, o que pode ser identificado quando o CV for maior que $60 \%$ a $70 \%$.

\section{Considerações sobre o uso do método}

Deve-se lembrar que este método para avaliar a adequação aparente da ingestão de nutrientes pelo indivíduo tem as mesmas fontes de erro dos dados dietéticos: subestimação ou superstimação do consumo alimentar, erros de memória, acurácia das tabelas de composição de alimentos, entre outros ${ }^{11}$.

Reconhece-se que a utilização da variação intrapessoal obtida em estudos populacionais, representando a variação intrapessoal de todos os indivíduos amostrados no mesmo estágio de vida e gênero, pode trazer vieses para a estimativa da adequação, já que esta variação é extremamente pessoal e, portanto, diferente de indivíduo para indivíduo7,11. Mesmo assim, o "Subcomitê para Uso e Interpretação das DRIs" recomenda utilizar esta medida, já que dificilmente períodos curtos de inquérito dietético poderiam representar toda a gama de valores de ingestão do nutriente². Até o presente momento, não há estudos que permitam ter uma avaliação confiável da variância intrapessoal, obtida de registros dietéticos individuais. No Brasil, não há estudo de base populacional publicado com medidas repetidas de inquéritos alimentares. Assim, apesar dos inegáveis avanços para corrigir as distorções geradas quando se utilizava a RDA, deve-se ser extremamente cauteloso ao interpretar os resultados obtidos.

Há pouca informação disponível acerca da verdadeira variabilidade das necessidades dos nutrientes entre os indivíduos no mesmo estágio de vida e gênero ${ }^{2}$. O coeficiente assumido de 10\% ou $15 \%$ para niacina, por exemplo, pode não ser um estimador confiável da variabilidade da necessidade, resultando na perda de acurácia da estimativa.

\section{Limitações do método}

Há situações em que esta abordagem não é apropriada: a) quando a ingestão diária observada não for normal (ou simetricamente distribuída). Neste caso, observa-se que o CV é maior que $60 \%$ a $70 \%$. Nesta situação estão os seguintes nutrientes: carotenóides, vitamina $A$, vitamina $C$, vitamina $E$, vitamina $\left.B_{12}, e, b\right)$ quando a distribuição das necessidades do nutriente não for normal ou simétrica. Neste caso se encontram as necessidades de ferro de mulheres em idade fértil, devido às perdas de ferro decorrentes da menstruação.

Nestes casos, não há alternativa oferecida para avaliação da ingestão do nutriente².

\section{CONSIDERAÇÕ ES FINAIS}

A avaliação de consumo de alimentos é importante como indicador indireto do estado nutricional. Mais que isso, avaliar a ingestão de nutrientes de forma qualitativa e quantitativa é fundamental para promover-se mudanças no comportamento alimentar, e, com isso, colaborar para diminuir os riscos de adoecer, ou para melhorar a qualidade de vida.

Os métodos propostos para utilização das DRI devem ser utilizados com bastante cautela. A avaliação dietética deve ser baseada em critérios metodológicos bem definidos e, principalmente em bom senso, para que, no final, tanto o diagnóstico quanto a orientação dietética sejam confiáveis.

\section{REFER Ê NCIAS}

1. Christakis G. Nutritional assessment in health programs. Am J Publ Health 1973; 63(Suppl): 1-82.

2. Institute of Medicine. Dietary Reference Intakes: applications in dietary assessment. Washington DC; 2000. 306p. 
3. Dietary Reference Intakes. Nutr Rev 1997; 55(9):332-4.

4. Liu K, Stamler J, Dyer A, Mckeever J, Mckeever P. Statistical methods to assess and minimize the role of intra-individual variability in obscuring the relationship between dietary lipids and serum cholesterol. J Chron Dis 1978; 31:399-418.

5. Tarasuk V, Beaton GH. Statistical estimation of dietary parameters: implications of patterns in within-subject variation - a case study of sampling strategies. Am J Clin Nutr 1992; 55:22-7.

6. Nusser SM, Carriquiry AL, Dood KW, Fuller WA. A semiparametric transformation approach to estimating usual daily intake distribuitions. J Am Stat Assoc 1996; 91:1440-9.

7. Beaton GH. Approaches to analysis of dietary data: relationship between planned analyses and choice of metodology. Am J Clin Nutr 1994; 59 Suppl:253s-61s.
8. Willet WC. Nutritional epidemiology. 2nd ed. Oxford: Oxford University Press; 1998. 514p.

9. Sempos $C T$, Looker $A C$, Johnson $C L$, Woteki $C E$. The importance of withing -person variability in estimating prevalence. In: Monitoring Dietary Intakes. New York: Springer-Verlag; 1991. p.99-109.

10. Barr SI, Murphy SP, Poos MI. Interpreting and using the dietary reference Intakes in dietary assessment of individual and groups. J Am Diet Assoc 2002; 102:780-8.

11. Armstrong BK, White E, Saracci R. Principles of exposure measurement in epidemiology. 2nd ed. Oxford: Oxford University Press; 1995. 351p.

12. Tarasuk VS, Brooker AS. Interpreting epidemiologic studies of diet-disease relationships. J Nutr 1997; 127:1847-52.

Recebido para publicação em 23 de setembro de 2002 e aceito em 24 de abril de 2003. 